\title{
In focus in HCB
}

\author{
Douglas J. Taatjes ${ }^{1}$ · Jürgen Roth ${ }^{2}$
}

Accepted: 22 April 2021 / Published online: 11 May 2021

(c) The Author(s), under exclusive licence to Springer-Verlag GmbH Germany, part of Springer Nature 2021

In this May issue of the journal, for the edification of our readers, we present relevant information concerning "Read and Publish" (Springer Compact) agreements, and highlight three Original Articles and one Short Communication describing (1) the expression and localization of cholesterol transporter proteins throughout the human hair cycle; (2) the characterization of the epithelial-to-mesenchymal transition and the relationship of primary cilia in this process in human urothelial cancers; (3) the effect of ovarian hormones and human choriogonadotropin on the polarity of human endometrial cells prior to implantation; and (4) the effects of epidermal maturation and UV-irradiation on calcium-activated chloride-associated protein rCLCA2 in cultured rodent keratinocyes and intact skin. We wish you good reading!

\section{Read and publish (Springer compact) - benefits for authors and readers}

Histochemistry and Cell Biology, published by Springer Nature, is referred to as a "hybrid journal", indicating that it is a subscription-based journal with optional "Open Choice" (Springer Open Choice) for Open Access publication. In the original version of this publishing model, the Open Access publication fee has to be covered by the authors. The recently implemented "Read and Publish" (Springer Compact) agreements permit authors to publish Open Access at no cost to themselves, provided their institution has a Read and Publish agreement. To find out which institutions have such an agreement in place, please access:

https://www.springer.com/gp/open-access/springer-openchoice/springer-compact/.

Douglas J. Taatjes

douglas.taatjes@med.uvm.edu

1 Department of Pathology and Laboratory Medicine, Larner College of Medicine, University of Vermont, Burlington, VT 05405, USA

2 University of Zurich, 8091 Zurich, Switzerland
Through the Read and Publish agreement, the Open Access publication fee is covered by the author's institution. Additional benefits for authors include:

1. Higher visibility and greater impact of their manuscript since Open Access articles are accessed 4 times more often on average, and cited 1.6 more times on average than those published without Open Access in Springer hybrid journals.

2. Easy compliance with mandates from funding agencies, since many now require Open Access publication of manuscripts resulting from their funding; some funding agencies may even consider this compliance when assessing future grant applications.

3. Last but not least, the copyright remains with the authors since most Open Choice articles are published under the liberal Creative Commons Attribution 4.0 International (CC BY) license.

\section{After all, greasy hair is not that bad}

Cholesterol is important for hair growth and cycling, and dysregulation of cholesterol homeostasis has been implicated in various hair disorders (Palmer et al. 2020). In their present work, Palmer and colleagues (2021) applied immunofluorescence to determine the cellular expression and localization of various cholesterol transport proteins-ABCA1, ABCG1, ABCA5 and SCARB1-in human hair follicles throughout the hair cycle. In addition, filipin was used as a stain for free cholesterol. Cultured outer root sheath (ORS) keratinocytes were used for Western blot and gene expression analyses and cholesterol efflux assays. The following is an excerpt from the multitude of beautifully illustrated results reported by the authors. The ubiquitous cholesterol efflux transporter ABCA1 showed a distinct staining pattern with higher expression in the epithelial compartment compared with the mesenchymal connective tissue sheath (CTS) in anagen hair follicles (Fig. 1). The ORS of the isthmus displayed the 


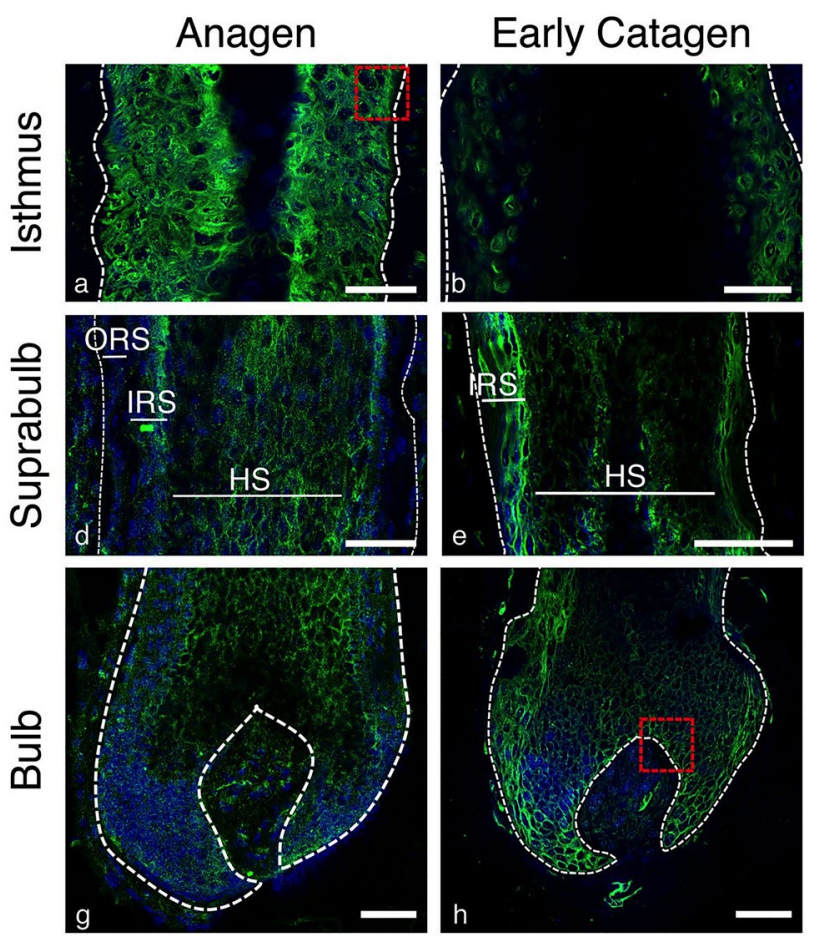

Fig. 1 Immunofluorescence localization of ABCA1 (green) in human anagen and early catagen hair follicles. DAPI counterstaining (blue). From Palmer et al. (2021)

highest staining intensity, with a polarized distribution in the basal ORS. The immunostaining within the inner root sheath was indistinct, whereas it was membranous in the hair shaft cuticle. Differential distribution patterns were also observed during catagen. 3-hydroxy-3-methylglutaryl-coenzyme A reductase is the enzyme responsible for the rate-limiting step in cholesterol synthesis. During anagen, intense immunostaining for the enzyme was found in the matrix, dermal papilla and ORS (being highest within the isthmus) with lower levels in the IRS and the hair shaft. Immunostaining in the mesenchymal connective tissue sheath was low to absent. It was concluded that the widespread expression of 3-hydroxy-3-methylglutaryl-coenzyme A reductase across the hair cycle points to the capability of hair shafts for de novo cholesterol synthesis. This conclusion was supported by the filipin staining for free cholesterol. In a nutshell, the authors demonstrated the capacity of human hair follicles for cholesterol transport and trafficking.

\section{A primary cilia EMT response in bladder cancer...}

Epithelial-to-mesenchymal transition (EMT) is a wellknown process involved in multiple aspects of tumor progression (Zhang and Weinberg 2018). EMT is driven by a variety of signaling pathways, including Hedgehog (Hh). Hh signaling is interestingly dependent upon a primary cilia-type mechanism (Bangs and Anderson 2017), and has been shown to be involved in the carcinogenic mechanisms of various types of cancer (Eguether and Hahne 2018). Iruzubieta et al. (2021) have now investigated the potential role of primary cilia-driven Hh signaling in the progression of bladder cancers. Urothelial tumors, the most common form of bladder cancer, are classified into non-muscle invasive bladder cancers (NMIBC) and muscle invasive bladder cancers (MIBC) (Humphrey et al. 2016). In their study, utilizing tissue samples from normal and both subclasses of urothelial cancers, Iruzubieta et al. (2021) used immunohistochemistry and immunofluorescence staining employing antibody markers for epithelial cell and mesenchymal cell phenotypes, Hh signaling pathway proteins, and cilia (Fig. 2). Furthermore, they performed a detailed

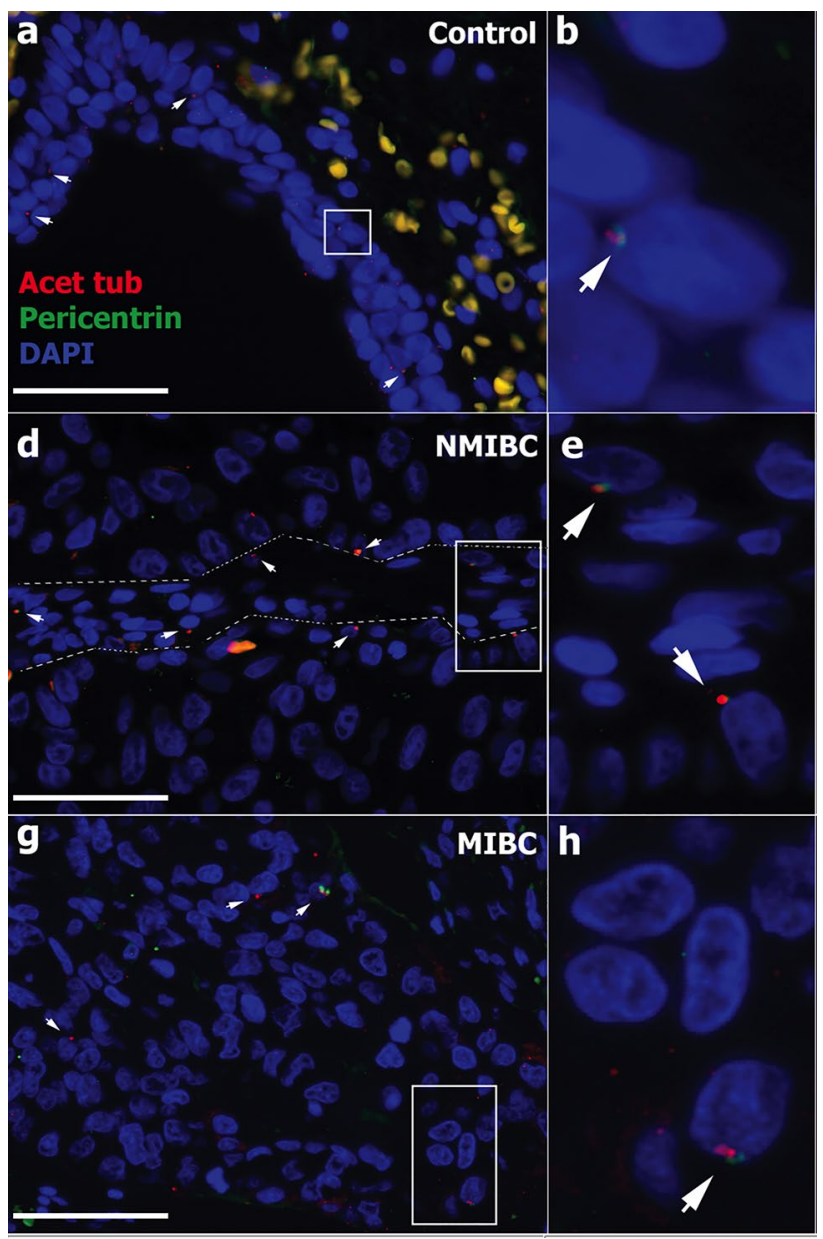

Fig. 2 Primary cilia in healthy urinary bladder (control), Non-Muscle Invasive Bladder Cancer (NMIBC) and Muscle Invasive Bladder Cancer (MIBC). Acetylated tubulin (red immunofluorescence) marks ciliary axoneme, while Pericentrin (green immunofluorescence) labels centrioles and, consequently, basal bodies. DAPI counterstaining (blue). From Iruzubieta et al. (2021) 
transmission electron microscopic analysis of urothelial cells and tumors to characterize the ultrastructural features of the cells. Their immunohistochemical results demonstrated the occurrence of EMT in both types of bladder cancers, as well as the presence of primary cilia in cells from both normal and bladder tumor samples. The electron microscopy results detailed the ultrastructural features of the tumor cells, and described for the first time the presence of primary cilia in healthy normal and cancerous bladder cells. Overall, their study added further details concerning the possible roles of the Hh signaling pathway and primary cilia in the process of urothelial cancer progression.

\section{Implanting the idea of steroid hormone influence on epithelial cell polarity}

The implantation of the human embryo into the endometrium represents a striking instance of non-cancerous tissue invasion. Indeed, just prior to embryo implantation, the human endometrium undergoes a complicated remodeling process, involving alterations in the polarity of epithelial cells related to the redistribution of junctional complex proteins including desmosomal and adherens junction proteins. In this regard, in earlier published work, Buck and colleagus used immunofluorescence microscopy to investigate the localization and distribution of endometrial epithelial junction proteins during the human menstrual cycle (Buck et al. 2012), and further demonstrated the great utility of creating and using endometrial spheroids as a model system for studying human embryo implantation (Buck et al. 2015). In their current investigation, Buck et al. (2021) continue their use of the endometrial spheroid model to investigate the effect of steroid hormones and human choriogonadotropin on the polarity-inducing localization of cellular adhesion proteins. They created spheroid cultures from the Ishikawa human endometrial cell line, treated them with ovarian steroids or human choriogonadotropin, and then performed multilabel immunostaining followed by wide-field light microscopic imaging. They found that treatment of the spheroids with progesterone, medroxy-progesterone acetate, or human choriogonadotropin resulted in a redistribution of the desmosomal plaque protein Dsp-1 to the basolateral membrane, while the zonula occludens protein ZO-1 remained in the apical membrane (Fig. 3). Likewise, the same hormone treatments resulted in a redistribution of the extracellular matrix adhesion protein $\alpha 6$-integrin to the lateral membrane; staining of human tissue samples from different stages of the menstrual cycle confirmed this redistribution of $\alpha 6$-integrin. Thus, these results extend and confirm the hypothesis that a hormone-effected decrease in epithelial cell polarity is required for the receptivity of the endometrium for embryo
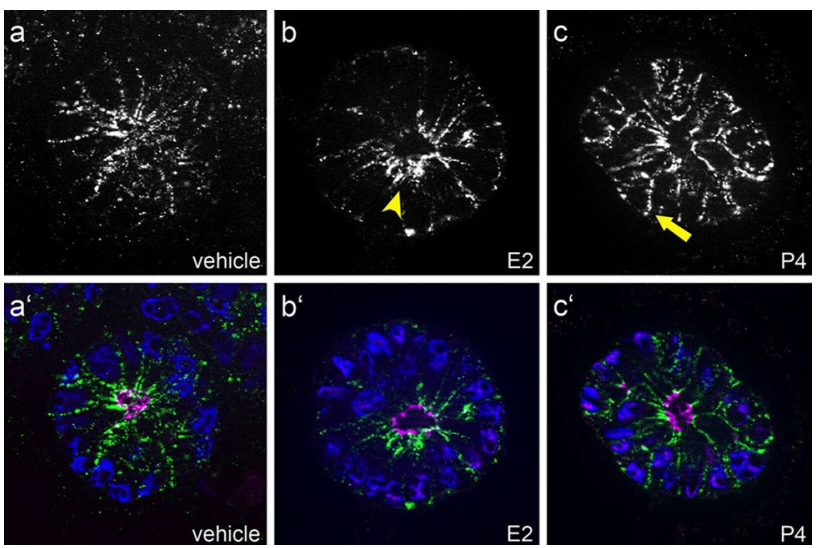

Fig. 3 Influence of ovarian steroid hormones on the localization of desmoplakin 1 (Dsp-1, green) in gland-like endometrial epithelial spheroids. E2 estradiol, $P 4$ progesterone, Zonula occludens protein-1 (ZO-1, red) and DAPI (blue). From Buck et al. (2021)

implantation. Moreover, the authors demonstrate the great value and utility of using cellular spheroids for 3D tissue models in human reproductive research, as has also been shown recently for other tissues such as lung (Cunniff et al. 2021) and diseases such as cancer (Huch and Koo 2015).

\section{A chloride channel-associated protein keeps keratinocytes quiet}

An insult that damages the skin barrier requires a quick response to restore its structure and function. Among the various repair components and mechanisms, different chloride channels may be involved since they play a role in keratinocyte migration, proliferation, and differentiation (Dong et al. 2015; Guo et al. 2016; Pan et al. 2015) as well as tumor suppression (Zhang et al. 2013). The activity of the chloride channels seems to be regulated by various chloride channel accessory proteins (Patel et al. 2009) that are also present in epidermal keratinocytes (Braun et al. 2010; Connon et al. 2004). Through their regulatory effect, they can modulate cell proliferation and apoptosis, and via their integrin-binding domains, they can promote cell adhesion and control migration and invasion. However, distinct functional
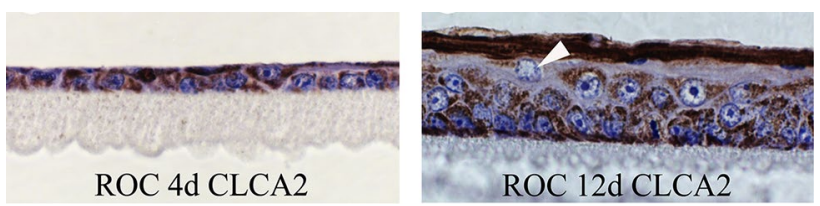

Fig. 4 Rat epidermal keratinocytes stratify and differentiate when grown in organotypic cultures (ROC) for 4 or 12 days and express CLCA2 (brown). From Hämäläinen et al. (2021) 
species-related differences among the various chloride channel accessory proteins have been reported. Therefore, Hämäläinen et al. (2021) have analyzed the expression and possible function of the rat calcium-activated chloride channel-associated protein rCLCA2 in cultured rat epidermal keratinocytes and correlated their findings with the mouse homolog in mouse skin (Fig. 4). They observed high and stable expression levels of rCLCA2 mRNA and protein in cultured rat epidermal keratinocytes and in organotypic cultures throughout the different stages of epidermal maturation. Through siRNA-mediated silencing, the authors showed that rCLCA2 facilitates UV-induced apoptosis. However, this condition did not significantly influence the keratinocyte migration in a scratch wound assay. Furthermore, they observed that a single UV irradiation resulted in a modest down-modulation of $r C L C A 2$ mRNA, with a duration of at least 7 days. In addition, the number of UV irradiation caused apoptotic cells was reduced by rCLCA2 silencing. The authors concluded that rCLCA2 functioning through its proapoptotic action prevents survival of damaged cells and that rCLCA2 appears to be a UV-irradiation target gene.

\section{References}

Bangs F, Anderson KV (2017) Primary cilia and mammalian Hedgehog signaling. Cold Spring Harbor Perspect Biol 9(5):a028175. https:// doi.org/10.1101/cshperspect.a0218175

Braun J, Bothe MK, Mundhenk L, Beck CL, Gruber AD (2010) Murine mCLCA5 is expressed in granular layer keratinocytes of stratified epithelia. Histochem Cell Biol 133:285-299

Buck VU, Windoffer R, Leube RE, Classen-Linke I (2012) Redistribution of adhering junctions in human endometrial epithelial cells during the implantation window of the menstrual cycle. Histochem Cell Biol 137:777-790. https://doi.org/10.1007/ s00418-012-0929-0

Buck VU, Gellerson B, Leube RE, Classen-Linke I (2015) Interaction of human trophoblast cells with gland-like endometrial spheroids: a model system for trophoblast invasion. Hum Reprod 30:906916. https://doi.org/10.1093/humrep/dev011

Buck VU, Kohlen MT, Sternberg AK, Rösing B, Neulen J, Leube RE, Classen-Linke I (2021) Steroid hormones and human choriogonadotropin influence the distribution of alpha6-intgegrin and desmoplakin 1 in gland-like endometrial epithelial spheroids. Histochem Cell Biol. https://doi.org/10.1007/s00418-020-01960-z

Connon CJ, Yamasaki K, Kawasaki S, Quantock AJ, Koizumi N, Kinoshita S (2004) Calcium-activated chloride channel-2 in human epithelia. J Histochem Cytochem 52:415-418
Cunniff B, Druso JE, van der Velden JL (2021) Lung organoids: advances in generation and 3D-visualization. Histochem Cell Biol 155:301-308. https://doi.org/10.1007/s00418-020-01955-w

Dong J, Jiang X, Zhang X, Liu KS, Zhang J, Chen J, Yu MK, Tsang LL, Chung YW, Wang Y, Zhou WL, Chan HC (2015) Dynamically regulated CFTR expression and its functional role in cutaneous wound healing. J Cell Physiol 230:2049-2058

Eguether T, Hahne M (2018) Mixed signals from the cell's antennae: primary cilia in cancer. EMBO Rep 19(11):e46589. https://doi. org/10.15252/embr.201846589

Guo R, Pan F, Tian Y, Li H, Li S, Cao C (2016) Down-regulation of $\mathrm{ClC}-3$ expression reduces epidermal stem cell migration by inhibiting volume-activated chloride currents. J Membr Biol 249:281-292

Hämäläinen L, Bart G, Takabe P et al (2021) The calcium-activated chloride channel-associated protein rCLCA2 is expressed throughout rat epidermis, facilitates apoptosis and is downmodulated by UVB. Histochem Cell Biol. https://doi.org/10.1007/ s00418-021-01962-5

Huch M, Koo BK (2015) Modeling mouse and human development using organoid cultures. Development 142(18):3113-3125

Humphrey PA et al (2016) The 2016 WHO classification of tumors of the urinary system and male genital organs-part B: prostate and bladder tumours. Eur Assoc Urol 70(1):106-119. https://doi.org/ 10.1016/j.eururo.2016.02.028

Iruzubieta P, Castiella T, Monleon E, Berga C, Munoz G, Junquera C (2021) Primary cilia presence and implications in bladder cancer progression and invasiveness. Histochem Cell Biol. https://doi. org/10.1007/s00418-021-01965-2

Palmer MA, Blakeborough L, Harries M, Haslam IS (2020) Cholesterol homeostasis: links to hair follicle biology and hair disorders. Exp Dermatol 29:299-311. https://doi.org/10.1111/exd.13993

Palmer MA, Smart E, Haslam IS (2021) Localisation and regulation of cholesterol transporters in the human hair follicle: mapping changes across the hair cycle. Histochem Cell Biol. https://doi. org/10.1007/s00418-020-01957-8

Pan F, Guo R, Cheng W, Chai L, Wang W, Cao C, Li S (2015) High glucose inhibits $\mathrm{ClC}-2$ chloride channels and attenuates cell migration of rat keratinocytes. Drug Des Dev Ther 9:4779-4791

Patel AC, Brett TJ, Holtzman MJ (2009) The role of CLCA proteins in inflammatory airway disease. Annu Rev Physiol 71:425-449

Zhang Y, Weinberg RA (2018) Epithelial-to-mesenchymal transition in cancer: complexity and opportunities. Front Med 12:361-373. https://doi.org/10.1007/s11684-018-065606

Zhang JT, Jiang XH, Xie C, Cheng H, Da Dong J, Wang Y, Fok KL, Zhang XH, Sun TT, Tsang LL, Chen H, Sun XJ, Chung YW, Cai ZM, Jiang WG, Chan HC (2013) Downregulation of CFTR promotes epithelial-to-mesenchymal transition and is associated with poor prognosis of breast cancer. Biochim Biophys Acta 1833:2961-2969

Publisher's Note Springer Nature remains neutral with regard to jurisdictional claims in published maps and institutional affiliations. 